Meta

Journal des traducteurs

Translators' Journal

\title{
Pour une formation universitaire « sui generis » du traducteur
}

\section{Roger Goffin}

Volume 16, numéro 1-2, mars 1971

Actes du colloque international de linguistique et de traduction.

Montréal, 30 septembre - 3 octobre 1970

URI : https://id.erudit.org/iderudit/002938ar

DOI : https://doi.org/10.7202/002938ar

Aller au sommaire du numéro

Éditeur(s)

Les Presses de l'Université de Montréal

ISSN

0026-0452 (imprimé)

1492-1421 (numérique)

Découvrir la revue

Citer cet article

Goffin, R. (1971). Pour une formation universitaire « sui generis » du traducteur. Meta, 16(1-2), 57-68. https://doi.org/10.7202/002938ar d'utilisation que vous pouvez consulter en ligne. 


\title{
Pour une formation universitaire «sui generis» du traducteur
}

\author{
RÉFLEXIONS SUR CERTAINS ASPECTS MÉTHODOLOGIQUES \\ ET SUR LA RECHERCHE SCIENTIFIQUE DANS LE DOMAINE DE LA TRADUCTION
}

Traduire a toujours été une tâche ardue et pourtant, la traduction a presque de tout temps laissé la porte ouverte aux dilettantes dont la ronde n'a cessé d'asphyxier la profession. Il n'y a pas bien longtemps l'on confiait une traduction à une secrétaire bilingue, car on tenait la traduction pour un simple artisanat ou une affaire de praticien appliquant, avec plus ou moins de bonheur, un ensemble de recettes. Certes, pour mener à bien son ouvrage, le traducteur doit connaître un certain nombre de «ficelles» et faire cuvre de haute fidélité et de dépendance. Certes, le traducteur a parfois poussé la fidélité jusqu'à la transposition abécédaire de l'original et a confondu traduction et psittacisme. Par ailleurs, la traduction était regardée comme une œuvre d'érudit et le grand public éprouvait à l'égard de son auteur, un peu en marge, un sentiment d'admiration et de suspicion, de respect et de circonspection. De même que l'on naissait poète, on naissait traducteur.

On comprend dès lors aisément que la traduction ne s'enseignait pas : ou bien la congénialité et l'affinité d'écriture avec l'auteur guidaient le traducteur, ou bien seules comptaient les connaissances linguistiques et l'expérience pratique ${ }^{1}$.

La traduction n'était pas pour autant bannie de l'enseignement des langues dispensé dans les facultés, toutefois elle y tenait un rôle ancillaire. Pendant des siècles, l'université a regardé la traduction comme un simple exercice d'acquisition de la langue, et non comme une discipline autonome. La dichotomie entre étude de la philologie et étude de la traduction tenait au fait que seuls les textes littéraires et la langue fortement individualisée des bons auteurs (peut-être parce qu'elle échappe à toute codification) retenaient l'attention des philologues, et non les textes scientifiques et diplomatiques soumis à la sagacité des traducteurs. Si d'aventure philologues et traducteurs se sont trouvés devant le même texte, ils $\mathrm{y}$ ont cherché des éléments différents.

Les données du monde contemporain ont bouleversé les vieilles conceptions que se faisaient les philologues bon teint de ce que l'on appelle désormais l'《activité traduisante ». Il y a d'abord l'universalisation des informations, il y a

1. Jean Amsler, traducteur français de G. Grass, dans une lettre adressée le 2 février 1969 , à Mlle Kratzmuller : "Se mettre dans la peau de l'auteur, dans le cas de Grass, cela m'a été facile par une affinité naturelle. » 
ensuite la scientifisation de tous les domaines de l'activité humaine, il y a, enfin, la révolution pédagogique dans l'enseignement des langues.

C'est devenu un truisme que d'affirmer que la traduction est une activité essentielle de notre époque, au cours de laquelle science et technique ont fait explosion. L'importance, capitale aujourd'hui, des échanges internationaux dans les secteurs diplomatiques et scientifiques a donné à la traduction un éclat nouveau. La multiplicité et la complexité croissante des sujets traités imposent des normes de qualité sans cesse plus sévères. L'activité traduisante acquiert ainsi une réelle spécificité, à telle enseigne qu'une profession autonome est née, encore mal circonscrite parfois, mais dont certains s'efforcent, avec acharnement, de préciser les contours et de définir les voies d'accès.

On constate par ailleurs l'irruption de la pensée scientifique dans la plupart des domaines qui lui étaient restés jusqu'ici étrangers. On sait que, depuis 1946, l'idée de soumettre les formes matérielles du langage aux méthodes d'analyse électronique n'a cessé de hanter l'esprit des scientifiques. Ce sont eux d'ailleurs qui ont, en quelque sorte, forcé les philologues à porter leur effort sur la recherche de critères objectifs applicables à la traduction. C'est des exigences de la pratique que sont nées, pour une large part, les théories récentes.

Reste la révolution pédagogique. Dès l'instant où les pédagogues se sont rendus à l'évidence que la traduction n'était pas une méthode d'enseignement des langues qui répondait aux besoins pratiques et que l'on pouvait, par une heureuse conjoncture biologique, connaître très bien deux langues sans pourtant être en mesure de les traduire, le problème de la traduction a pris une dimension nouvelle. La connaissance des langues n'apparaît plus que comme un outil, indispensable certes, qu'il faut apprendre à manier avec une dextérité accrue, mais qui ne constitue qu'un facteur parmi beaucoup d'autres dans l'opération traduisante.

D'ancillaire, la traduction ne devint pas d'un coup magistrale. Son émancipation fut lente. Pourtant, les plus récents ouvrages consacrés à la traduction semblent vouloir faire de celle-ci une branche autonome de la linguistique. Les théoriciens multiplient les enquêtes pour découvrir les indices de son autarcie et lui conférer le statut de discipline exacte. Ils cherchent, au-delà de l'empirisme des procédés artisanaux ou de l'inspiration congéniale et des intuitions heureuses, à démonter la traduction en un mécanisme de causes et d'effets, complexe et ramifié, mais transparent. Ils ne cessent de s'atteler à définir les lois et règles qui régissent l'opération traduisante, laquelle s'érige progressivement en science.

Dans son Introduction à la théorie de la traduction, A. V. Fedorov explique l'importance pratique de la traduction «par la nécessité de s'appuyer sur des principes objectifs et fondés scientifiquement qui excluraient l'arbitraire subjectif du traducteur et interdiraient d'en référer à l'intuition comme justification de cet arbitraire ${ }^{2} \gg$.

Dernière-née des sciences du langage, cette discipline cherche encore, c'est évident, son nom, ses axiomes et ses méthodes. Le problème de la dénomination reste évidemment lié à celui de la délimitation de la notion; comme toujours,

2. A. V. Fedorov, Introduction à la théorie de la traduction, traduit du russe par $\mathbf{R}$. Deresteau et A. Sergeant (mémoire de licence présenté en 1969 à l'Ecole supérieure de traducteurs et d'interprètes de l'Etat à Bruxelles), 1953, p. 27. 
l'heureuse découverte d'un terme approprié pourrait sans doute faciliter les progrès des recherches. En français, "traductiologie » et «translatologie» sont des néologismes plus ou moins bien frappés, en allemand, Übersetzungswissenschaft déjà courant, est né dans le sillage de Sprachwissenschaft et par analogie à celle-ci. En néerlandais, vertaalkunde ne s'est pas encore imposé, mais tirera parti de son presque homonyme taalkunde, quant à science of translation, il commutera bientôt, semble-t-il, avec translatology.

Cette discipline cherche aussi ses axiomes, et sur ce point plusieurs écoles s'affrontent, car la question est d'importance. La traduction est-elle une opération linguistique ou est-elle une opération paralinguistique?

Fedorov part du postulat suivant : «Puisque la traduction est toujours liée à la langue et implique une étude de celle-ci, puisqu'elle demande toujours, sous l'angle linguistique, une étude du caractère des rapports entre deux langues, la théorie de la traduction en tant que branche spéciale de la philologie apparaît avant tout comme une discipline linguistique ${ }^{3} . \gg$

L'étude de la traduction est tout naturellement centrée sur celle du langage humain. Or, au cours des quinze dernières années, certains aspects de la linguistique ont profondément évolué; les linguistes portent leur attention non plus sur le développement ou l'aspect diachronique des langues, mais sur leur fonctionnement réel et ont, à cette fin, introduit des méthodes de caractère logico-mathématique. Comme le montre Revzin ${ }^{4}$, la linguistique a été jusqu'ici taxinomique; après avoir observé un nombre suffisant de faits de langage, le linguiste les intégrait dans un système de relations de manière à en dégager une certaine ordonnance. Le travail de rassemblement et de classement des faits prenait appui sur une méthode empirique comparative de nature inductive. L'étude de la traduction était fondée sur l'analyse comparée, sinon contrastive, bilatérale de deux langues à travers l'original et la traduction et ce dans un but normatif, celui d'élaborer des critères qualitatifs permettant de juger la traduction. Or, E. Sapir notamment a démontré qu'aucune des ordonnances proposées n'était satisfaisante et c'est cet échec des méthodes de classification traditionnelles dans l'étude comparée des langues qui a été un des éléments moteurs pour l'élaboration d'une linguistique formalisée.

Grâce à l'élan que leur a donné le structuralisme, les linguistes s'efforcent de soustraire la linguistique et la traduction à toute appréciation subjective et d'établir une espèce d'algèbre du langage dans le dessein de construire des modèles formels de fonctionnement ou schémas abstraits. Les observations systématisées des structuralistes américains font apparaître les phrases et les syntagmes comme des ensembles hiérarchisés représentables par des arborescences bifurcantes. Cette recherche se fait par la méthode déductive : «La théorie de la traduction est une science qui dispose de ses méthodes et catégories spécifiques et qui affronte ses problèmes propres; elle doit donc s'échafauder essentiellement par voie de déduction ${ }^{5} . »$

3. A. V. Fedorov, Introduction à la théorie de la traduction, p. 14.

4. I. Revzin, les Modèles linguistiques, Paris, 1968, traduit du russe par Y. Gentilhomme, cité d'après la recension de G. L. dans : le Linguiste, 6 (1968), p. 20-21.

5. I. Revzin et $\mathrm{V}$. Rosenzweig, les Fondements de la traduction générale et de la traduction automatique, traduit par G. Chantrain (mémoire de licence), 1967-1968, p. 47. 
Aux yeux des praticiens, les modèles fonctionnels apparaissent parfois comme des édifices préfabriqués, stériles et abstraits, détachés de la contemplation quotidienne des textes. Devant l'infinie diversité des textes avec lesquels ils se collettent, les vétérans de la traduction ne croient guère à la schématisation de leur activité, alléguant qu'il n'est pas de solution passe-partout et que, dans la multitude des situations possibles, il s'en présente toujours une pour déjouer les meilleurs schémas. Même du côté des informaticiens-linguistes, il y a des sceptiques. C'est ainsi que S. Perschke affirme : "sous leur forme actuelle, les algorithmes de la linguistique mathématique, les grammaires transformationnelles n'ont pas enrichi, par rapport à la grammaire traditionnelle, la description de la langue ; ils semblent au contraire l'avoir appauvrie ${ }^{6}$ ».

On constate en effet que bon nombre de théoriciens se cantonnent dans une spéculation intellectuelle un peu vaine lorsqu'ils élaborent leurs schémas formalistes ou formalisés. Au contraire, l'analyse minutieuse du processus traduisant devrait, elle, permettre de donner une assise scientifique aux méthodes du traducteur. De même que l'analyse du processus de création poétique est devenue une préoccupation essentielle des poètes modernes (Valéry, Eliot, Mallarmé), de même l'analyse de l'acte traduisant doit se trouver au cour même de toute réflexion théorique sur la traduction. Nos considérations ne s'appliqueront qu'à la traduction de textes pragmatiques dans lesquels la langue est conçue pour l'expression objective de faits observables et où la forme s'efface toujours au profit de l'objet.

On connaît le schéma classique de la traduction fondé sur la théorie de la communication. À l'aide du système sémiologique et sémantique de la langue de départ (LD), le locuteur $A$ transmet une communication $C^{1}$ relative à une situation de la réalité. Le traducteur, dans son rôle de premier récepteur, est saisi du message $C^{1}$ et procède à une translation en se référant ou non à la réalité, après quoi, en se servant du système de la langue d'arrivée (LA), il forme une nouvelle communication $C^{2}$, laquelle est reçue par le destinataire $B$, qui établit à son tour une corrélation entre $C^{2}$ et la réalité à l'aide du système LA.

Ce schéma fondamental doit être précisé dans sa partie centrale, laquelle comporte trois phases : a) l'analyse du texte (le décodage) ; b) le changement de code; $c$ ) la synthèse (recodage). Dans son rôle de premier récepteur, le traducteur appréhende le message par la lecture, c'est-à-dire par la perception visuelle répétitive de signifiants qui agissent comme des signaux. L'aberration du message reçu sera d'autant plus grande que le traducteur est éloigné de la connaissance spécialisée de la matière traitée et de la terminologie utilisée. Même s'il s'est familiarisé avec la technologie évoquée par le texte, le traducteur ne peut se représenter dans sa totalité les intentions de l'auteur, car à un même mot, ou groupe de mots, il fera correspondre, en fonction de son expérience antérieure, une partie différente du réseau de connaissances. Tandis qu'au cours de la lecture, les parcelles de réalité contenues dans le texte défilent à toute allure sur l'écran de son esprit, il édifie, pierre par pierre, un contexte de fait assez flou, à partir de son expérience antérieure. Toute expérience de la réalité

6. S. Perschke, "Traduction automatique, possibilités et limites", Bulletin Euratom, 2 (juin 1967), p. 58. 
laisse dans les centres nerveux une trace de nature biochimique semblable à l'empreinte laissée par un chasseur traversant une forêt. La perception sensorielle d'un mot par la lecture crée, elle aussi, dans le système nerveux une trace ou engramme déterminé. Si l'objet ou le concept est présenté avec le mot, un lien se constitue entre le mot et ledit concept : l'engramme du concept, qui est la trace sous-tendant la fixation du souvenir, s'associe à l'engramme du mot ${ }^{\top}$.

Lorsque les mots et les matières lui sont familiers, le traducteur établit une correspondance immédiate et totale entre le message et les engrammes. II comprend parce qu'il reconnaît. Dans le cas inverse, si le traducteur ignore tout des mots et des matières, l'établissement d'une correspondance message-engrammes n'est plus possible. Il ne comprend pas.

Dans la plupart des cas, le message peut être rattaché d'une façon assez lâche à l'engramme grâce à une analyse poussée des rapports internes du texte. Le traducteur ne recevra pourtant du texte que des signifiés amoindris ${ }^{8}$.

Le passage à la réalité ou encore «aux choses et phénomènes» équivaut à un passage sur les engrammes existants. Cette référence directe à la « réalité » ou au désigné est-elle indispensable ? Que faut-il penser de la boutade de Bertrand Russell qui affirmait : "No one can understand the meaning of cheese until he has a non linguistic acquaintance of it $\gg$. Si l'on sait la diversité des textes soumis à la sagacité du traducteur technique d'aujourd'hui, force est de constater que ce dernier doit bien souvent aborder des sujets dont il n'a pas toujours une connaissance intime : l'électronique, la métallurgie du plutonium, les combustibles à ailettes utilisés dans les réacteurs, le soudage par aluminothermie, la radiostérilisation des mouches tsé-tsé mâles, tout cela est sa pâture hebdomadaire, et, faute d'être tout à la fois biologiste, généticien, électronicien, bromatologue et neutronicien, il lui faudra fourbir d'autres armes. Toutefois, la non-compréhension paralysante initiale n'est pas rédhibitoire. Le traducteur dispose de moyens plus ou moins extensibles qu'il devra mettre en œuvre pour activer ou réactiver sa connaissance des mots et des matières. La perception visuelle répétitive lui permet, au cours de lectures successives, de tirer des enseignements du contexte linguistique. La réactivation des mots passe par l'analyse de ce que Bühler a appelé «le contexte synsémantique ${ }^{9} \gg$. Ce dernier comporte successivement $: a$ ) l'analyse terminologique; b) l'analyse syntagmatique; et c) l'analyse syntactique.

A. L'analyse terminologique du texte comporte plusieurs possibilités ${ }^{10}$ : a) en ce qui concerne la monosémie

$1^{\circ}$ La recherche du monosème de la LA équivalent au monosème de la LD. Le passage peut s'effectuer sans recours au dénoté, et la correspondance être totale. C'est le cas pour les appellatifs, les noms d'éléments, les numéraux, les unités.

7. P. V. Hendrickx, "Talen leren en leren vertalen », Revue des langues vivantes, 4 (1969), p. 405-414.

8. R. Goffin, "La terminologie multilingue et la syntagmatique comparée au service de la traduction technique ", Babel, 3 (1968), p. 132-142; voir aussi : META, XIV, 4 (déc. 1969), compte rendu de Irène V. Spilka, p. 231-235.

9. K. Bühler, Sprachtheorie, die Darstellungsfunktion der Sprache, Stuttgart, 1965, t. 2, p. 154ss.

10. Voir à ce propos l'excellente étude de O. Kade, «Zufall und Gesetzmässigkeit in der Übersetzung ", Fremdsprachen, Leipzig, Beiheft Nr. 1, 1968, 128 p. 
Si la plupart des transplutoniens ont fait l'unanimité sur leur nom : le berkélium (97), le californium (98), l'einsteinium, voire le mendélévium sont internationaux, l'élément 102 a été baptisé nobélium, mais il y a là dispute autour du nom. Les Suédois disent l'avoir découvert en 1957, les Américains en 1958, les Russes quelques années plus tard; ceux-ci prétendent même que les laboratoires soviétiques sont les seuls à avoir effectué les expériences convaincantes. Ils suggèrent pour leur part de rebaptiser le nobélium et de lui donner, sans esprit chauvin, le nom de joliotium ${ }^{11}$;

$2^{\circ} \mathrm{La}$ recherche de monosèmes synonymes dans la LA équivalents au monosème de la LD. Ce passage s'effectue sans recours au dénoté. Dans les vocabulaires techniques en gestation, celui de l'atome par exemple, on peut constater un foisonnement de lexèmes synonymes. Ainsi, «facteur de désavantage » et «facteur de flux neutronique» sont utilisés pour désigner disadvantage factor; "état normal » est synonyme de «état fondamental » pour désigner ground state; «paramètre de ralentissement» est synonyme du calque anglais « décrément logarithmique moyen de l'énergie »;

$3^{\circ} \mathrm{La}$ recherche d'un monosème dans la LA équivalent au monosème LD ; toutefois les champs sémantiques sont différents, il y a correspondance partielle. En français, le terme anglais burn up (= combustion nucléaire) est souvent employé en physique nucléaire pour burn up fraction (= taux d'épuisement) et specific burn up (= combustion massique);

$4^{\circ} \mathrm{La}$ recherche d'un syntagme, voire d'une expression qui sert d'équivalent à un monosème sans correspondant linguistique. Dans le vocabulaire de la conduite des réacteurs, derating (syn. stretch out) est le procédé qui consiste à commencer par différer un nouveau chargement du réacteur et à maintenir le réacteur en fonctionnement pendant un court laps de temps à l'aide d'éléments partiellement épuisés et de puissance réduite.

\section{b) en ce qui concerne la polysémie}

$1^{\circ}$ Réduction de la polysémie initiale en monosémie grâce à la clef terminologique, semblable à la clef musicale, puis recherche de l'équivalent monosémique sans passage au dénoté. Plasma prend au milieu du $\mathrm{xx}^{\mathrm{e}}$ siècle le sens de matière gazeuse très chaude constituée d'ions positifs et d'électrons, électriquement neutre et très conductrice. Malgré la température toujours très élevée, on parle de «plasma» froid. Divergence est employé en mathématiques ou physique pour décrire une situation de deux lignes ou de deux rayons qui vont en s'écartant. La «divergence » nucléaire est l'établissement de la réaction en chaîne dans un réacteur ;

$2^{\circ}$ Réduction de la polysémie complexe du terme stripping, dont l'usage est différent des deux côtés de l'Atlantique :

$\begin{array}{llll}\text { U.K. } & \text { U.S.A. } & \text { France } & \text { Domaines } \\ \text { stripping } & \text { scrubbing } & \text { lavage } & \text { (chimie) } \\ \text { backwashing } & \text { stripping } & \text { réextraction } & \text { (solvent extraction) } \\ \text { stripping } & \text { stripping } & \text { stripage } & \text { (réacteur nucléaire) }\end{array}$

11. R. Goffin, "Réflexions sur la terminologie de l'atome », Equivalences, 1 (1970), p. 15-27. 
Le traducteur technique peut ainsi souvent associer des monosèmes stéréotypés ou polysèmes réduits en monosèmes, de LD à LA, sans faire intervenir réellement le dénoté. Dans la traduction, le référent ne fait pas du tout partie du schéma. Le passage direct, presque automatique, aboutit à une équivalence totale. En fait, le traducteur n'a pas le choix entre plusieurs unités possibles dans la LA, il doit reconnaître et utiliser les relations d'équivalence présentes dans le système ${ }^{12}$.

L'analyse terminologique, pour importante qu'elle soit, ne constitue qu'un aspect de l'analyse du contexte synsémantique. La conception du mot-récipient ou de la langue-catalogue s'appuie sur une simplification selon laquelle le monde serait un arsenal d'objets où chaque objet serait pourvu d'une plaquette amovible qu'il suffirait de changer pour passer d'une langue à l'autre.

B. L'analyse syntagmatique doit retenir toute l'attention du traducteur pour une double raison : a) le syntagme est la formation de base de textes scientifiques, appelée à une production infinie, $b$ ) le syntagme est souvent différent d'une langue à l'autre. La langue scientifique moderne use volontiers d'un type de composition qui consiste en une collocation de plusieurs lexèmes en un seul complexe, joints ou non par un ligament. C'est le syntagme au sens saussurien : «le syntagme se compose toujours de deux ou de plusieurs unités consécutives. Placé dans un syntagme, le terme n'acquiert sa valeur que parce qu'il est opposé à ce qui précède ou ce qui suit ou à tous les deux ${ }^{13}$.» Syntaxiquement, son comportement est identique à celui des monèmes avec lesquels il peut commuter, et sémantiquement, il se réfère à une seule notion, à une idée unique comme le dit Bailly ${ }^{14}$.

En français, nous pouvons, suivant la nature grammaticale et l'ordre des lexèmes, distinguer trois types de syntagmes : a) le syntagme asyndétique, formé d'un substantif suivi d'un substantif, du type compteur cloche (bell counter tube) ou verre dosimètre (glass dosimeter) ; b) le syntagme épithétique, formé d'un substantif et d'un adjectif dit de relation, du type énergie nucléaire, exemple : force nucléaire, potentiel nucléaire, droit nucléaire; c) le syntagme avec joncteur, c'est la synapsie de Benvéniste, type de composition qui consiste en une unité de lexèmes reliés par des joncteurs ou ligaments synaptiques.

Par rapport au composé allemand, la synapsie se caractérise par l'emploi de joncteurs pour marquer la nature syntaxique de la liaison, l'ordre des membres et l'absence d'article devant le déterminant. En allemand comme en français, on retrouve le caractère unique et constant du signifié. Dans la synapsie, nécessairement analytique, $70 \%$ des synapsies présentent le joncteur de, 20\% les joncteurs $\grave{a}$ ou par, sous. Il serait d'ailleurs intéressant, mais cela nous conduirait trop loin, de décomposer les syntagmes dont un des éléments est le même mot. Nous constatons d'emblée que la même construction syntaxique (dans le composé allemand, le ligament synaptique est nul) peut correspondre à des faits différents de connaissance, la connaissance ne s'intégrant pas dans le système linguistique.

12. Etiemble, le Jargon des sciences, Paris, 1968, p. 71. L'auteur propose le mot "étripage ", parent d'étrépage, qui est l'action de défricher à l'aide d'un étrèpe.

13. F. de Saussure, Cours de linguistique générale, Paris, 1964, p. 170.

14. C. Bailly, Linguistique générale et linguistique française, 4e éd., Berne, paragr. 141, p. 94. 
La compétition entre les synapsies (dose de rayonnement) et les syntagmes épithétiques correspondants (dose radiologique) n'est pas moins ardente que celle qui, dans d'autres cas, oppose les syntagmes épithétiques (protection radiologique, radiographie neutronique) aux néologismes morphologiques (radioprotection, neutrongraphie). Par ailleurs, «polymérisation par rayonnement » commute avec « radiopolymérisation», «sensibilité aux rayonnements » avec « radiosensibilité », "mal des rayons» (maladie radiologique) avec « radiotoxémie ». Ainsi le préfixe radio entame-t-il une carrière nouvelle avec radio-exposition, radioélément, radio-activimètre (le trait d'union n'est là que pour éviter une collision de deux voyelles), radiobiologie, radiocarottage, radiométallographie, radioprotecteur, radiorésistance.

Vu la complexité de certains syntagmes, le traducteur qui aura consulté le dictionnaire multilingue de conception traditionnelle en sera pour sa peine. Il ne trouvera son salut que dans les vocabulaires contextuels de la lignée des glossaires phraséologiques publiés par les bureaux de terminologie. Les termes y sont insérés dans un contexte qui permet de préciser leur champ terminologique, et qui fournit bon nombre de données lexicographiques complémentaires. L'emploi de ces structures caractéristiques permettra au traducteur de se rapprocher de son idéal : faire une traduction qui se lit comme un original. L'expérience a démontré que ce sont bien souvent les verbes, propositions et adjectifs entourant le substantif technique qui constituent autant de chausse-trapes pour le traducteur.

C. L'analyse synsémantique comporte enfin l'analyse syntactique. La grammaire transformationnelle a montré l'existence, dans toutes les langues, d'un nombre limité de structures profondes à partir desquelles les formes plus élaborées se construisent par transformation. L'analyse syntactique est le processus analytique qui consiste à réduire les structures élaborées en structures de base. Or, on a constaté qu'au niveau des structures profondes les langues s'accordent beaucoup plus qu'au niveau des structures élaborées. De plus, le passage de la structure profonde de la LD à la LA se fait, dans $70 \%$ des cas, par transposition simple. Dans $30 \%$ des cas, le traducteur devra avoir recours à la métataxe qui est, au sens où l'entend L. Tesnière, un changement structural ; elle peut aller du simple appel à une catégorie grammaticale différente (transposition ou grammaticalisation) jusqu'à la transformation complète de l'ordonnance structurale avec changement du nœud central. Tesnière distingue d'ailleurs : la métataxe simple, l'interversion double ou multiple des actants ( $I$ miss you $\sim$ vous me manquez), le changement de diathèse du verbe ${ }^{15}$.

Dans une étude consacrée à l'enseignement des langues, Gyula Eidei estime à $25 \%$ environ le nombre de phrases types dont la structure profonde diffère en allemand et en hongrois ${ }^{16}$. C'est sur ce type de structure profonde que l'enseignement devra construire ses exercices.

Le traducteur scientifique doit, plus fidèlement encore que son collègue traducteur littéraire, suivre le tracé et la chronologie de la phrase et reproduire avec le même soin l'essentiel et le secondaire. Une soumission intelligente à

15. L. Tesnière, Eléments de syntaxe structurale, Paris, 1959 , p. $283 \mathrm{ss}$.

16. G. Eidei, "Zum Problem des Übersetzens im Fremdsprachenunterricht », Deutsch als Fremdsprache, 3 (1970), p. 225. 
l'écriture de l'original lui permet de dégager les points nodaux du message. Ces points nodaux, il doit les chercher non pas dans les substantifs, comme on le croit trop souvent quand il s'agit de textes techniques, mais dans le nœud verbal qui constitue le noud central de la phrase. Comme le fait remarquer Tesnière, c'est abusivement que les grammairiens allemands ont baptisé le substantif Hauptwort ( $=$ mot principal).

Fouquet a, un jour, comparé la structure de la phrase à un trousseau de clés où les dépendants directs du verbe (actants et circonstants) seraient représentés par les différentes clés et le nœud verbal par l'anneau qui les retient ensemble. Cette conception de la phrase, je dirai volontiers verbocentrique ou «verbipète », permet de dégager les structures profondes ou les «kernels » dont parle E. A. Nida ${ }^{17}$.

La (ré)activation des matières se fait à l'aide de la documentation. Un texte n'est jamais soumis à la traduction sans être accompagné d'un certain nombre de documents de référence qui servent essentiellement de documents de travail. Ce sont des articles spécialisés sur le sujet traité ou très apparenté à celui-ci, des rapports similaires. La lecture de ces documents permet au traducteur de prendre la mesure de l'orientation et de la teneur de son texte. Il fera ainsi une incursion dans le monde scientifique pour acquérir de son texte une compréhension suffisante. À mesure que le traducteur compulse son dossier, les idées et les mots s'approchent pêle-mêle, pas très perceptibles encore à la conscience. On peut se demander jusqu'où il poussera son incursion. Tout bien considéré, il devra s'arrêter en chemin, car un autre impératif le guette : le délai! Plutôt que de chercher fébrilement à tout propos le passage du dossier où il croit avoir aperçu une tournure équivalente, il devra se jeter, tête baissée, dans sa traduction, la dicter puis rectifier son brouillon en y insérant tel ou tel vocable ou tournure, car une fois la traduction dictée, les expressions qu'elle contient restent mieux gravées dans la mémoire parce qu'elles ont exigé un effort de réflexion.

$\mathrm{Si}$ nous nous sommes attardé, un peu longuement peut-être, sur l'analyse du processus de traduction, c'est pour montrer quelles sont les perspectives qu'elle ouvre à la didactique de la traduction. La recherche pédagogique au niveau de l'enseignement supérieur a été quasi inexistante au cours des dernières années et il faut bien admettre que, malgré les réformes qui ont récemment secoué les bastilles de l'université, l'enseignement dispensé dans les facultés reste étroitement lié à la langue des bons auteurs et à l'aspect esthétique et diachronique du langage.

Dans la société d'aujourd'hui, l'activité traduisante a acquis une réelle autonomie à telle enseigne qu'il est désormais devenu indispensable de donner une formation sui generis à ceux qui se destinent à la carrière de traducteur. En effet, alors que naguère on ne choisissait pas la traduction pour métier, aujourd'hui beaucoup de jeunes entrent en traduction, ils font "acte de traduction», pour reprendre le mot de P. F. Caillé ${ }^{18}$.

Ils espèrent tous qu'une voie royale leur sera ouverte, une voie qui mène aux grandes organisations internationales. Nous savons qu'il y a beaucoup d'appelés

17. E. A. Nida, Theory and Practice of Translation, Leiden, 1969, p. 39.

18. P. F. Caillé, "Traduire, c'est choisir ", Babel, 1 (1967), p. 11. 
et peu d'élus, mais la responsabilité des enseignants à l'égard des jeunes, de l'université et d'eux-mêmes, est considérable. Et de fait, les critiques adressées à ces écoles spécialisées sont vives venant des praticiens et de l'université. Pour les uns, les cours feraient une part trop large à la théorie, verseraient dans l'abstraction, accorderaient trop d'importance à l'aspect historique. Pour les facultés, ces écoles se limiteraient à la transmission d'un savoir ou à l'apprentissage d'une technique sans corps de doctrine. Or, à quoi bon créer des institutions spécialisées, échafauder des programmes si les méthodes d'enseignement, elles, demeurent classiques?

Pour conclure, je suggérerai quelques principes dont on pourrait utilement s'inspirer :

a) Il importe avant tout que, dans les écoles spécialisées, la traduction soit enseignée comme une discipline exacte et comme une science qui n'est point accumulation de faits, mais savoir organisé et pensé. L'étudiant doit prendre conscience du fait que le métier auquel il se prépare s'exerce non plus de manière empirique, mais selon des règles précises qu'on l'aidera à découvrir par des exercices appropriés. La priorité sera donnée à l'apprentissage des processus logiques d'analyse et à l'étude des structures profondes des langues étudiées. Les exercices de traduction viseront à faire confronter la structure profonde de phrases sémantiquement identiques dans la langue de départ et d'arrivée. Ces exercices de «traduction confrontative» (de confrontation de structures) se distinguent des exercices de traduction de caractère morphologique ou lexicologique. Les exercices seront choisis de manière à éviter que, lors de la traduction d'une structure profonde LD, l'étudiant puisse utiliser dans LA une structure identique à $\mathrm{LD}$; en effet, dans ce cas, il donnerait automatiquement en LA la structure calquée sur LD. Il faudra ensuite lui montrer comment faire manœuvrer un système de phrases et lui apprendre à maîtriser les procédés de la métataxe. Le traducteur doit aussi prendre conscience des systèmes sémiologiques que constituent les langues étudiées et du système hiérarchisé de marques qui désignent les procédures de référence au réel ;

b) L'enseignement devrait résolument porter sur l'acquisition d'un vocabulaire fondamental des sciences et techniques, du type «vocabulaire général d'orientation scientifique » (VGOS), et sur l'étude d'une langue de spécialité. L'exploration d'une langue de spécialité doit être l'occasion de faire comprendre à l'étudiant les liens qui existent entre un vocabulaire spécialisé et le système conceptuel qu'il reflète, de faire comprendre l'interaction qui se produit entre le vocabulaire de spécialité et le vocabulaire usuel, d'étudier selon quelles lois le jargon des sciences ombrage la langue contemporaine, enfin de passer en revue les néologismes morphologiques, sémantiques et syntagmatiques, les faux amis, etc.;

c) L'enseignement doit prévoir une initiation aux méthodes documentaires et à la constitution de fichiers linguistiques (Sprachkartei). Il faudra éveiller le goût pour la documentation scientifique bilingue destinée à l'exploitation terminologique pour que le traducteur puisse rapidement s'imprégner des sujets et en acquérir une compréhension suffisante. L'aptitude à se documenter seul devra lui être inculquée par le mémoire de terminologie; 
d) L'école devra permettre à l'étudiant d'entamer son processus d'adaptation à la vie avant sa sortie, d'où l'organisation de stages dans les milieux professionnels ;

$e)$ Il faut prévoir une initiation aux méthodes d'utilisation de l'ordinateur qui est en voie de devenir un instrument indispensable pour la mise en mémoire de données terminologiques et pour la consultation automatique.

Dans toutes les écoles supérieures de traducteurs, il faut que l'enseignement soit indissolublement associé à la recherche. La recherche liée à l'enseignement de la traduction pourrait aller vers la recherche fondamentale de nature abstraite et théorique. Elle viserait à construire un corps de doctrine cohérent permettant d'interpréter, au moyen de lois plus générales, les phénomènes mis en jeu dans la traduction (neurolinguistique). Elle serait caractérisée par l'établissement de schémas théoriques. Toutefois, l'élaboration des théories ne peut se faire qu'à partir des données patiemment accumulées par les observateurs et collecteurs de données. C'est là que se dessine une autre forme de recherche, la recherche au service de, d'application.

Le mémoire de terminologie, imposé par la plupart des instituts, semble s'inscrire parfaitement dans l'esprit et le contexte de cet enseignement. Disons d'emblée qu'au moment de proposer une liste de sujets, le professeur fera bien de tendre l'oreille du côté des praticiens ou, de préférence, du côté des bureaux de terminologie qui seraient invités à soumettre des thèmes de recherches propres. Ce serait là de la recherche appliquée ou fondamentale orientée, dans la mesure où l'utilité pratique serait le facteur essentiel du choix du sujet.

Le mémoire, à la fois monographie et glossaire, conçu dans un esprit pragmatique, permettra peut-être, à sa manière, d'éviter le regrettable hiatus existant, en Europe, entre l'enseignement supérieur et l'industrie et de rapprocher l'étudiant de son employeur éventuel.

S'il fallait maintenant conclure par quelques paroles édifiantes, on pourrait notamment répéter que, combattant à sa manière l'ignorance, le traducteur doit considérer sa tâche comme un moyen d'aiguiser son sens critique et de parfaire ses connaissances. Toute activité professionnelle qui n'est pas purement répétitive comporte une certaine part de recherche : le traducteur sera, sa vie durant, un chercheur. S'il ne sera jamais un Pic de la Mirandole dans les spécialités dont relèvent les textes qui sont soumis à sa sagacité, du moins pourra-t-il ressembler au chercheur persévérant - et la persévérance est fille de l'humilité - persuadé qu'il n'existera dans la société de demain que dans la mesure où il s'efforce sans cesse de maîtriser un savoir infini qui se dérobe constamment devant lui.

ROGER GOFFIN

\section{QUESTIONS}

M. Vinay : Quand on parlait avec des universitaires français, il n'était pas question de technique de la traduction, c'était je pense, un don du Saint-Esprit. Peut-être cette attitude change-t-elle maintenant? Je le souhaite et d'autant plus que nous pouvons bénéficier, comme vous l'avez très bien fait remarquer, de nouvelles techniques dans la terminologie et particulièrement dans la syntaxe. Il est certain que des travaux comme ceux de Chomsky peuvent être applicables à l'enseignement de la traduction.

M. Prévost : Est-ce que vous croyez qu'un traducteur, et là je parle d'un traducteur dont l'employeur, par exemple, est un organisme d'État, doit être orienté, dans le sens propre 
du terme, vers un secteur traductionnel défini ? C'est-à-dire qu'il devrait être orienté par son employeur vers une spécialisation définitive, même si la polyvalence du traducteur doit en souffrir. Souvent cette polyvalence est la base même de la fonction professionnelle du traducteur dans l'organisme qui l'emploie.

Réponse : Il me semble avoir montré qu'au niveau de l'enseignement, il était important d'initier le traducteur, le candidat traducteur, à un secteur général de la science, lui faire comprendre les mécanismes du vocabulaire et de la syntaxe de textes techniques, de lui enseigner non pas la terminologie spécifique de la physique des plasmas par exemple, mais de lui donner un certain nombre de connaissances exactes dans un contexte, des notions de chimie et de physique, mais je ne crois pas qu'on exige d'un traducteur diplômé qu'il soit spécialiste dans une technique particulière. II faudrait aussi, faire une conférence aux employeurs pour leur demander un peu d'indulgence. Nous, traducteurs généralistes, nous aimerions nous spécialiser dans tel on tel domaine, mais il nous faut quelques mois avant de pouvoir travailler dans un domaine particulier tel que les techniques nucléaires, par exemple. Je crois que nous devons rester des généralistes, nous ne pourrons jamais saisir le fond des problèmes scientifiques, c'est très décevant dans la profession. On a un petit vernis, on comprend mais on ne connaît pas, on ne peut pas émettre un avis sur un article sur la radiostérilisation des mouches mâles par exemple, mais on est peut-être en mesure de le traduire convenablement avec beaucoup de bonne volonté, de recherche et de persévérance, et du savoir-faire.

M. Dubuc : Si j'ai bien compris quand yous avez exposé votre théorie de l'enseignement de la traduction, vous avez insisté particulièrement sur la nécessité d'axer l'enseignement sur les différences de structures profondes entre les langues, est-ce que je pourrais vous demander de préciser un petit peu?

Réponse : La première phase doit viser non pas à résoudre les difficultés terminologiques dans un texte mais à montrer quelles sont les phrases, quelles sont les structures différentes, deux à deux bien entendu, et s'attacher à enseigner ces structures, quitte alors plus tard à y insérer les mots difficiles. Je veux donc réserver la première phase de l'enseignement de la traduction à l'analyse et à la traduction de phrases où il $\mathrm{y}$ a des contraintes et qui impliquent une modification de la structure. 